\title{
Automated Test Oracles for GUIs
}

by Atif Memon, Mary Soffa, Mary Pollack

This paper describes the use of an automated oracle to test the expected behavior of a GUI. The automation occurs both in the derivation of what the expected states of the GUI are and in the comparison of those expected states to the actual state of the GUI as it is being executed. The authors achieve this by first creating a model of the GUI's objects, properties, and actions. They then use this model to determine what the expected states of the GUI will be given any particular set of actions. The paper describes three different levels of testing that can be used to compare the expected states to the actual states of the GUI determining what to use is left up to the test designer.

After the authors lay out how to achieve the automated oracles, they give some performance results showing that the extra time needed to generate the automated test cases is not substantial. Consequently, they argue that even though there is a reasonable amount of human effort involved in creating the oracle, there is definitely an amortized benefit for the work that is done. I am not totally convinced of that argument since it was based on metrics gathered by testing a custom Notepad editor. It would take substantial effort to produce this oracle for ISVis especially considering that it is directly tied to an automated test case generator produced previously by the authors. All of that would have to be done from scratch if ISVis was to take advantage of this method. That being said, if it were in place, it would certainly be beneficial to future development and refactoring.

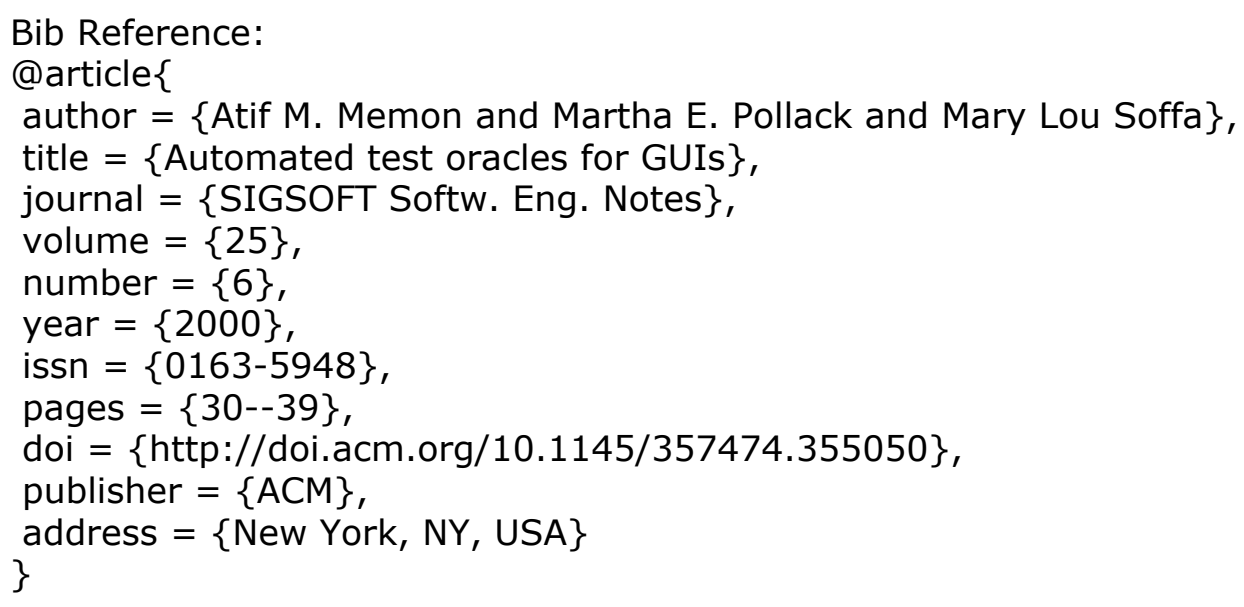

